\title{
Orbital mismatch boosting nematic instability in iron-based superconductors
}

\author{
Laura Fanfarillo, ${ }^{1}$ Lara Benfatto, ${ }^{2, *}$ and Belén Valenzuela ${ }^{3, \dagger}$ \\ ${ }^{1}$ CNR-IOM and International School for Advanced Studies (SISSA), Via Bonomea 265, I-34136 Trieste, Italy \\ ${ }^{2}$ ISC-CNR and Department of Physics, "Sapienza" University of Rome, P.le A. Moro 5, 00185 Rome, Italy \\ ${ }^{3}$ Instituto de Ciencia de Materiales de Madrid, ICMM-CSIC, Cantoblanco, E-28049 Madrid, Spain
}

(Received 30 July 2017; revised manuscript received 18 February 2018; published 22 March 2018)

\begin{abstract}
We derive the effective action for the collective spin modes in iron-based superconductors. We show that, due to the orbital-selective nature of spin fluctuations, the magnetic and nematic instabilities are controlled by the degrees of orbital nesting between electron and hole pockets. Within a prototypical three-pocket model the hole-electron orbital mismatch is found to boost spin-nematic order. This explains the enhancement of nematic order in FeSe as compared to 122 compounds, and its suppression under pressure, where the emergence of the second hole pocket compensates the orbital mismatch of the three-pocket configuration.
\end{abstract}

DOI: 10.1103/PhysRevB.97.121109

Understanding the origin of the nematic phase is one of the most challenging open issues in the field of iron-based superconductors (IBS). In these systems the structural transition from tetragonal to orthorhombic is accompanied (and often preempted) by a marked electronic anisotropy which suggests an electronic origin of the instability [1]. The original spin-nematic proposal $[2,3]$ focuses on the typical topology of the Fermi surface (FS) in pnictides, with hole-(h) like pockets at $\Gamma$ and electron-(e) like pockets at $\mathbf{Q}_{X}=(\pi, 0)$ and $\mathbf{Q}_{Y}=(0, \pi)$ in the $1 \mathrm{Fe}$ unit-cell notation. The underlying idea is that the nesting between $\mathrm{h}$ and e pockets favors the spin fluctuations at these two equivalent momenta. According to [2,3], a nematic phase emerges since the ellipticity of the e pockets induces an anisotropy of the paramagnetic spin fluctuations before the long-range magnetic order sets in, lowering the symmetry of the electronic response from $C_{4}$ to $C_{2}$. This appealing scenario is however challenged by the fact that nematicity is observed to be stronger or weaker in systems with similar band structure.

FeSe is a remarkable example. Here the undoped compound has a structural transition at $T_{S}=90 \mathrm{~K}$ which is only cut off below by the superconducting transition at $T_{c}=9 \mathrm{~K}$ [4]. The lack of magnetic order motivated alternative interpretations for nematicity as due to orbital ordering [5-10]. On the other hand, sizable spin fluctuations have been detected in FeSe as well $[11,12]$, triggering an intense investigation on the interplay between spin and orbital degrees of freedom [10,13-18]. Despite some interesting proposals [19-21], no consensus has been reached yet on the mechanism favoring nematicity in $\mathrm{FeSe}$ as compared to other systems, and leading to its suppression with external and internal pressure [22-24].

In this Rapid Communication we show that the spin-nematic scenario is able to discriminate topologically equivalent band structures once the original derivation $[2,3]$ is crucially revised accounting for the orbital character of the bands. On general grounds, the importance of the orbital content of the FS for the

\footnotetext{
*Corresponding author: lara.benfatto@ roma1.infn.it

${ }^{\dagger}$ Corresponding author: belenv@icmm.csic.es
}

low-energy spin fluctuations in IBS, pointed out in [13], has been recently discussed within several contexts [18,25-27]. Here we show that the orbital topology of the FS crucially affects the spin-nematic instability itself, which is controlled by the degree of orbital nesting, i.e., the relative orbital composition between the $h$ and e pockets involved in the spin-exchange mechanism. By projecting the general microscopic interaction [28-32] on the low-energy multiorbital model of [33], spin fluctuations at different $\mathbf{Q}$ vectors become orbital selective, i.e., they involve only specific orbitals [13] [see Fig. 1(a)]. As a result, also the interactions between spin modes beyond Gaussian level, responsible for the nematic instability, become renormalized by the orbital content of the $\mathrm{h}$ and e pockets. In particular, we find that orbital nesting can differentiate two topologically equivalent three-pocket models in which a single hole pocket is present at $\Gamma$. In the case of FeSe the relevant $\mathrm{h}$ pocket is the outer one [see Fig. 1(b)], and we find that its orbital mismatch with the e pockets boosts the nematic instability, while it is detrimental for magnetism. In contrast, in the 122 family the most relevant $\mathrm{h}$ pocket is the inner one [34-36], having opposite orbital character [see Fig. 1(b)]. In this case its good orbital nesting with the e pockets explains the robustness of the magnetic phase and the appearance of a nematic instability only in its proximity. Along the same reasoning, we argue that in FeSe the suppression of nematicity with internal or external pressure [22-24] can be ascribed to the emergence of the inner hole pocket, changing the FS orbital topology toward a more symmetric four-pocket model where nematicity can be easily lost.

We consider first a general four-pocket model with two $\mathrm{h}$ pockets at $\Gamma, \Gamma_{ \pm}$, and two e pockets at $X$ and $Y$, that can be easily adapted to describe different compounds among the 122 and 11 families. The kinetic part of the Hamiltonian is derived adapting the low-energy model considered in [33], where each pocket is described using a spinor representation in the pseudoorbital space $[18,33]$

$$
H_{0}^{l}=\sum_{\mathbf{k}, \sigma} \psi_{\mathbf{k} \sigma}^{l, \dagger} \hat{H}_{0}^{l} \psi_{\mathbf{k} \sigma}^{l},
$$




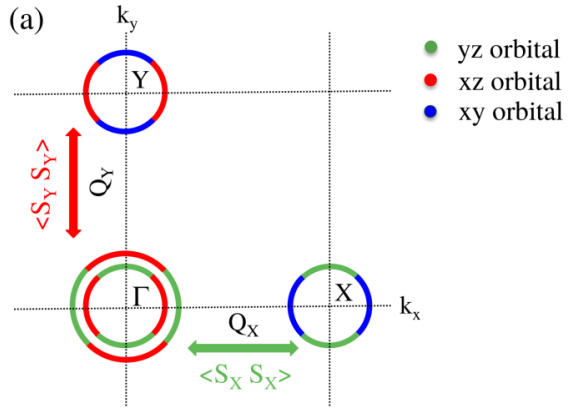

(b)

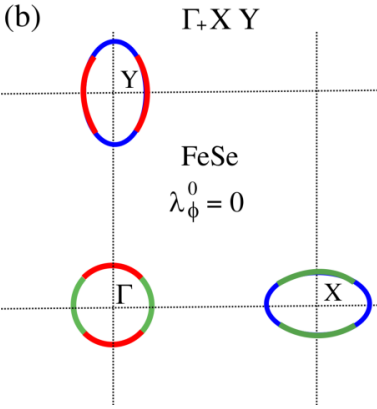

(c)

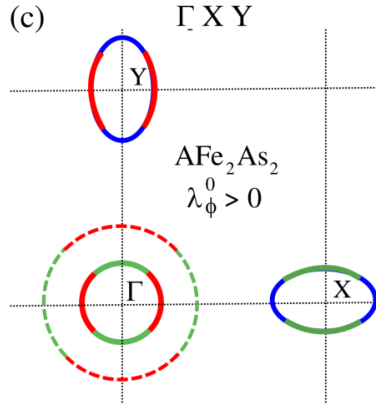

FIG. 1. (a) General sketch of the orbital content of the FS of the four-pocket model for IBS. The green/red arrows denote the OSSF, connecting $\mathrm{h}$ and e pockets at different momenta. (b) Sketch of FeSe: only the outer pocket is present. (c) Sketch of 122 systems: the outer pocket is much larger, so it can be neglected in first approximation. The orbital mismatch (matching) in panel (b) [(c)] is determined by the out-of-phase (in-phase) angular dependence of the $y z / x z$ orbital in the $h$ and $X / Y$ e pockets. $\lambda_{\phi}^{0}$ is the leading order value of the nematic coupling, see Eqs. (16) and (17).

with $\hat{H}_{0}^{l}=h_{0}^{l} \tau_{0}+\vec{h}^{l} \cdot \vec{\tau}, l=\Gamma, X, Y$, and $\tau$ matrices represent the pseudo-orbital spin. The spinors are defined as $\psi^{\Gamma}=$ $\left(c_{y z}, c_{x z}\right)$ and $\psi^{X / Y}=\left(c_{y z / x z}, c_{x y}\right)$. Diagonalizing $\hat{H}_{0}$ we find the dispersion relations $E^{l \pm}=h_{0}^{l} \pm h^{l}$ with $h^{l}=\left|\vec{h}^{l}\right|$. We introduce the rotation from the orbital to the band basis,

$$
\left(\begin{array}{l}
h_{+} \\
h_{-}
\end{array}\right)=\left(\begin{array}{cc}
u_{\Gamma} & -v_{\Gamma} \\
v_{\Gamma} & u_{\Gamma}
\end{array}\right)\left(\begin{array}{l}
c_{y z} \\
c_{x z}
\end{array}\right)
$$

with an analogous expression for the $X / Y$ pockets, provided that the corresponding orbital spinor is used. At $X / Y$ only the $E^{X / Y+}$ band crosses the Fermi level, so in the following we will use $e_{X / Y}$ for the corresponding fermonic operators dropping the + subscript.

The interacting Hamiltonian is given by

$$
H_{\text {int }}=-1 / 2 \sum_{\mathbf{q}^{\prime}} U_{\eta \eta^{\prime}} \vec{S}_{\mathbf{q}}^{\eta} \cdot \vec{S}_{-\mathbf{q}}^{\eta^{\prime}}
$$

with $\eta, \eta^{\prime}=y z, x z, x y$ denoting the orbital index. The interaction in the spin channel is defined as $U_{\eta \eta^{\prime}} \sim U \delta_{\eta \eta^{\prime}}+J_{H}(1-$ $\left.\delta_{\eta \eta^{\prime}}\right), U$ and $J_{H}$ being the usual Hubbard and Hund couplings. We consider only spin operators with intraorbital character $\vec{S}_{\mathbf{q}}^{\eta}=\sum_{\mathbf{k} s s^{\prime}}\left(c_{\mathbf{k} s}^{\eta \dagger} \vec{\sigma}_{s s^{\prime}} c_{\mathbf{k}+\mathbf{q} s^{\prime}}^{\eta}\right)$ with $\sigma_{s s^{\prime}}$ the Pauli matrices for the spin operator. This choice is motivated by the general finding that intraorbital magnetism is the dominant channel in IBS [28-32]. The relevant magnetic fluctuations occur at momenta $\mathbf{q}$ near $\mathbf{Q}_{X}$ or $\mathbf{Q}_{Y}$. At low energy we can project out the general interaction, Eq. (3), onto the fermionic excitations defined by the model (1). By using the rotation to the band basis, Eq. (2), one can then establish a precise correspondence between the orbital and the momentum character of the spin operators $\vec{S}_{X / Y}^{\eta} \equiv \vec{S}_{\mathbf{q}=\mathbf{Q}_{X / Y}}^{\eta}$

$$
\begin{aligned}
& \vec{S}_{X}^{y z}=\sum_{\mathbf{k}}\left(u_{\Gamma} h_{+}^{\dagger}+v_{\Gamma} h_{-}^{\dagger}\right) \vec{\sigma} u_{X} e_{X}, \\
& \vec{S}_{Y}^{x z}=\sum_{\mathbf{k}}\left(-v_{\Gamma} h_{+}^{\dagger}+u_{\Gamma} h_{-}^{\dagger}\right) \vec{\sigma} u_{Y} e_{Y},
\end{aligned}
$$

where we drop for simplicity the momentum and spin indices of the fermionic operators. It then follows that the interacting Hamiltonian, Eq. (3), reduces to

$$
H_{\text {int }}=-\frac{\tilde{U}}{2} \sum_{\mathbf{q}^{\prime}} \vec{S}_{X / Y}^{y z / x z} \cdot \vec{S}_{X / Y}^{y z / x z},
$$

where $\tilde{U}$ is the intraorbital interaction renormalized at low energy. As is clear from the above equation, it is the projection of the generic interaction Hamiltonian (3) onto the low-energy model (1) that generates orbital-selective spin fluctuations (OSSF). Indeed, since at low energy the $x z / y z$-fermionic states exist only around $Q_{Y} / Q_{X}$, it turns out that the spin operators $\vec{S}_{X}^{\eta}$ with $\eta \neq y z$ and $\vec{S}_{Y}^{\eta}$ with $\eta \neq x z$ are absent in Eq. (6), so that there are no terms involving the Hund's coupling. Once this correspondence has been established the derivation of the effective action is formally equivalent to the one used in the simplified band language [2]. One can decouple the interaction term, Eq. (3), by means of two vectorial Hubbard-Stratonovich (HS) fields $\vec{\Delta}_{X / Y}^{y z / x z}$ which will describe in what follows the collective electronic spin fluctuations. The effective action up to quartic order becomes

$$
\begin{aligned}
S_{\text {eff }}= & \left(\begin{array}{ll}
\Delta_{X}^{y z} & \Delta_{Y}^{x z}
\end{array}\right)\left(\begin{array}{cc}
\chi_{X}^{-1} & 0 \\
0 & \chi_{Y}^{-1}
\end{array}\right)\left(\begin{array}{c}
\Delta_{X}^{y z} \\
\Delta_{Y}^{x z}
\end{array}\right) \\
& +\left(\left(\begin{array}{ll}
\left.\Delta_{X}^{y z}\right)^{2} & \left(\Delta_{Y}^{x z}\right)^{2}
\end{array}\right)\left(\begin{array}{ll}
u_{11} & u_{12} \\
u_{12} & u_{22}
\end{array}\right)\left(\begin{array}{l}
\left(\Delta_{X}^{y z}\right)^{2} \\
\left(\Delta_{Y}^{x z}\right)^{2}
\end{array}\right)\right.
\end{aligned}
$$

Here $\chi_{X / Y}^{-1}=1 / U_{s}+\Pi_{X / Y}^{y z / x z}$, where $U_{s}$ is the effective interactions between low-energy quasiparticles, and $\Pi_{X / Y}^{y z / x z}$ is the propagator in the long-wavelength and zero-frequency limit:

$$
\begin{aligned}
& \Pi_{X}^{y z}=T \sum_{\mathbf{k}, i \omega_{n}} u_{\Gamma}^{2} u_{X}^{2} g_{+} g_{X}+v_{\Gamma}^{2} u_{X}^{2} g_{-} g_{X}, \\
& \Pi_{Y}^{x z}=T \sum_{\mathbf{k}, i \omega_{n}} v_{\Gamma}^{2} u_{Y}^{2} g_{+} g_{Y}+u_{\Gamma}^{2} u_{Y}^{2} g_{-} g_{Y} .
\end{aligned}
$$

$g_{i}\left(\mathbf{k}, i \omega_{n}\right)=\left(i \omega_{n}-E_{\mathbf{k}}^{i}\right)^{-1}$ are the Green's functions in the band basis, $i= \pm$ denotes the $\mathrm{h}$ bands, and $i=X, Y$ the electronic ones. The coefficients of the quartic part of the action in Eq. (7) are (see also [26])

$$
\begin{aligned}
& u_{11}=T \sum_{\mathbf{k}, i \omega_{n}}\left(u_{X}^{2} g_{X}\right)^{2}\left(u_{\Gamma}^{2} g_{+}+v_{\Gamma}^{2} g_{-}\right)^{2}, \\
& u_{22}=T \sum_{\mathbf{k}, i \omega_{n}}\left(u_{Y}^{2} g_{Y}\right)^{2}\left(v_{\Gamma}^{2} g_{+}+u_{\Gamma}^{2} g_{-}\right)^{2}, \\
& u_{12}=T \sum_{\mathbf{k}, i \omega_{n}} u_{X}^{2} g_{X} u_{Y}^{2} g_{Y} u_{\Gamma}^{2} v_{\Gamma}^{2}\left(g_{+}-g_{-}\right)^{2} .
\end{aligned}
$$



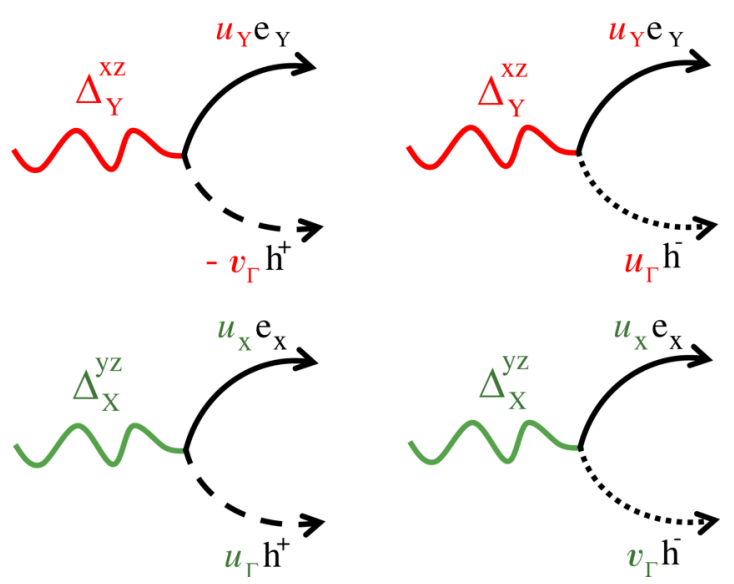

FIG. 2. Diagrammatic representation of the vertices connecting the HS fields to the fermionic operators. Wavy (red/green) lines denote the HS fields $\left(\Delta_{X / Y}^{y z / x z}\right)$, solid lines the excitations in the e pockets, and dashed/dotted lines excitations in the outer/inner h pocket. The $u_{l}, v_{l}$ coefficients account for the orbital component of each band, according to the low-energy projection (4) and (5).

As usual, the effective action is an expansion in powers of the HS fields. The coefficients of the $n$th power of the field is a loop with $n$ fermionic lines, leading to the product of two or four Green's functions in Eqs. (8), (9) and Eqs. (10)-(12), respectively. The vertices connecting $\Delta_{X / Y}^{y z / x z}$ to the band operators are depicted in Fig. 2. Using this correspondence, which follows from the projection (4) and (5) of the spin operators at low energy, one easily understands that the fermionic loops are weighted with the elements $u_{l}, v_{l}$ defining the orbital content of each band. The magnetic instability is controlled by the Gaussian part of the action, Eq. (7), and it occurs at the temperature where the inverse $Q_{X / Y}$ susceptibilities $\chi_{X / Y}^{-1}$ vanish. The nematic instability happens when the fluctuations along the $x$ and $y$ directions become inequivalent already above $T_{N}$. Since $u_{11}=u_{22}$ due to $C_{4}$ symmetry, the quartic part of the action, Eq. (7), can be simply diagonalized as

$$
S_{\text {eff }}^{(4)}=\lambda_{\psi} \psi^{2}+\lambda_{\phi} \phi^{2},
$$

where

$$
\begin{array}{ll}
\psi=\frac{1}{\sqrt{2}}\left(\left(\Delta_{X}^{y z}\right)^{2}+\left(\Delta_{Y}^{x z}\right)\right)^{2}, & \lambda_{\psi}=u_{11}+u_{12}, \\
\phi=\frac{1}{\sqrt{2}}\left(\left(\Delta_{X}^{y z}\right)^{2}-\left(\Delta_{Y}^{x z}\right)\right)^{2}, & \lambda_{\phi}=u_{11}-u_{12} .
\end{array}
$$

Notice that the tensorial form of the nematic order parameter proposed in Ref. [14] does not contain our result, Eq. (15), which in turn is dictated by the only possible non-Gaussian terms, Eq. (7), for the OSSF. From Eq. (13) one sees that a nematic instability is possible only for $\lambda_{\phi}<0$, when making $\langle\phi\rangle \neq 0$ lowers the energy of the system. However, while in Ref. [2] $\lambda_{\phi}$ is only controlled by the shape of the e pockets, we find that also the degree of orbital nesting plays an important role.

To make a first estimate of this effect we consider the simple case where the e/h pockets are perfectly nested circular FS, so that the orbital weights reduce to $u_{\Gamma}=u_{Y}=v_{X}=\cos \theta_{\mathbf{k}}$, $v_{\Gamma}=v_{Y}=u_{X}=\sin \theta_{\mathbf{k}}$, and the Green's functions can be writ- ten as $g_{X}=g_{Y}=g_{e}=\left(i \omega_{n}-\epsilon\right)^{-1}, g_{+}=g_{-}=g_{h}=\left(i \omega_{n}+\right.$ $\epsilon)^{-1}$, with $\epsilon=-\epsilon_{0}+\mathbf{k}^{2} / 2 m-\mu . \epsilon_{0}$ is the offset energy, $m$ the parabolic band mass, and $\mu$ the chemical potential. Within this approximation we can carry out explicitly the integration in Eqs. (8)-(12), showing that the differences between the various terms arise only from the angular integration of the product of the orbital weights. For what concerns the magnetic instability, the spin-fluctuation bubbles $\Pi_{X / Y}^{y z / x z}$, Eqs. (8) and (9), are both proportional to $\Pi_{e h}=T \sum_{\mathbf{k}, i \omega_{n}} g_{e} g_{h}$ that lead to the usual $\log$ divergence: $\Pi_{e h} \sim-N_{F} \log \omega_{0} / T$ where $N_{F}$ is the density of states and $\omega_{0}$ an upper cutoff [37]. On the other hand, the orbital renormalization of the $S_{\text {eff }}^{(4)}$ action is much more severe. Indeed, considering two hole pockets of the same size, one immediately finds from Eq. (12) that $u_{12}=0$. This leads to a large positive nematic eigenvalue $\lambda_{\phi}$ in Eq. (15), which prevents the occurrence of nematicity, in agreement with recent renormalization group studies on the four-pocket model [27].

To simulate the case of specific compounds we consider two three-pocket models in which a single hole pocket at $\Gamma$ is well nested with the elliptical e pockets: (a) The $3 p_{+}$model for FeSe [Fig. 1(b)], where only the outer pocket $\Gamma_{+}$crosses the Fermi level while the inner pocket $\Gamma_{-}$sinks below it before the nematic transition [18,24]. (b) The $3 p_{-}$model for 122 systems [Fig. 1(c)], where the outer pocket $\Gamma_{+}$is much larger than the electron ones, so it weakly contributes to the nesting [38,39]. These two models would be equivalent within the simplified band approach [2] but lead to different OSSF actions. As far as nematicity is concerned, we see that while the $u_{12}$ term in Eq. (12) is the same when only one of the two hole pockets is considered, the $u_{11}$ and $u_{22}$ terms pick up in a different way the orbital weights at $\Gamma$, allowing us to discriminate between the two cases.

(a) FeSe. As has been recently discussed in Ref. [18], the disappearance of the inner hole pocket in FeSe can be explained by the combined effect of spin-orbit coupling and OSSF shrinking mechanism. When only the $\Gamma_{+}$pocket is considered in Eqs. (10)-(12) all the coefficients of the quartic action become equal, so that at leading order $\lambda_{\psi}^{0}>0$ and $\lambda_{\phi}^{0}=0$. Following the same lines of [2], we then include at perturbative level the e-pockets ellipticity and the deviations from perfect nesting. Since the results are robust with respect to the latter perturbation [37], we discuss here only the dependence on the ellipticity parameter $\delta_{e}$. In this case, the eigenvalues of the quartic action turn out to be

$$
\lambda_{\psi}^{3 p_{+}}=3 \mathcal{K}(T), \quad \lambda_{\phi}^{3 p_{+}}=-\mathcal{K}(T) \frac{b \delta_{e}^{2}}{T^{2}}
$$

with $\mathcal{K}(T)=7 N_{F} \zeta(3) /\left(8^{3} \pi^{2} T^{2}\right)$. As one can see, as soon as a finite ellipticity is included, $\lambda_{\phi}<0$ at any temperature. This result is then analogous to the one found in the simplified band language of Ref. [2], and the nematic critical temperature is determined by the divergence of the full nematic susceptibility $\chi_{\text {nem }}=\int_{q} \chi_{X}^{2} /\left(1+\lambda_{\phi} \int_{q} \chi_{X}^{2}\right)$ [40]. On the other hand, the orbital mismatch between the $\mathrm{h}$ and e pockets realized in the case of FeSe is detrimental for the magnetic instability itself. Indeed, when only the $\Gamma_{+}$pocket is present the magnetic propagator in Eqs. (8) and (9) is reduced by a factor $1 / 8$ with respect to $\Pi_{e h}$ found in the simplified band language, since $\Pi_{X / Y}^{y z / x z} \sim \Pi_{e h} \int(d \theta / 2 \pi) \cos ^{2} \theta \sin ^{2} \theta=\Pi_{e h} / 8$ [37]. 

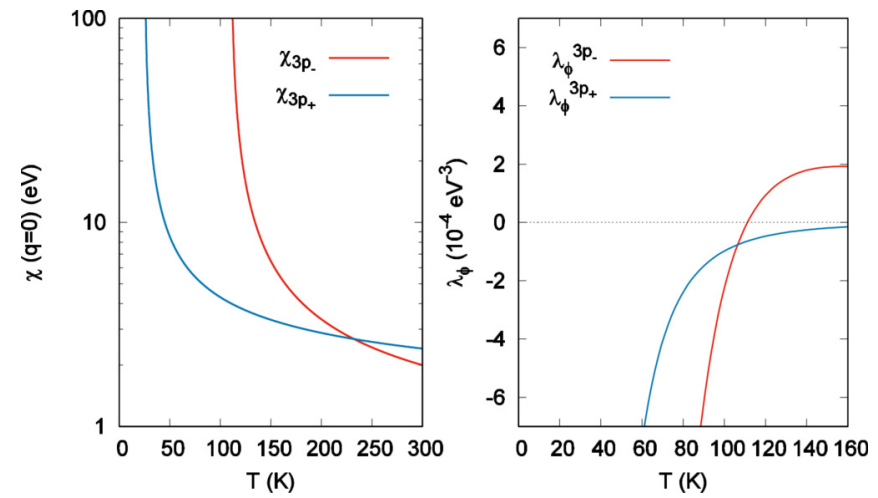

FIG. 3. (a) $\chi_{X / Y}^{y z / x z}(q=0)$ and (b) nematic eigenvalue $\lambda_{\phi}$ for the $3 p_{+}$and $3 p_{-}$model for the same set of band parameters (see text). Here $T_{\text {Néel }}=110,24 \mathrm{~K}$ for the $3 p_{-}, 3 p_{+}$model, respectively, while the change of sign of $\lambda_{\phi}$ for the $3 p_{-}$model occurs around $112 \mathrm{~K}$.

(b) 122 systems. In this case the good orbital nesting between the $\mathrm{h}$ and e pockets makes the $u_{11}$ term (10) much larger than the $u_{12}$ term (12), so that at leading order $\lambda_{\phi}^{0}$ in Eq. (15) is positive, preventing a nematic transition. Accounting for the ellipticity of the e pockets one finds

$$
\begin{aligned}
& \lambda_{\psi}^{3 p_{-}}=\mathcal{K}(T)\left(19-\frac{12 b \delta_{e}^{2}}{T^{2}}\right), \\
& \lambda_{\phi}^{3 p_{-}}=\mathcal{K}(T)\left(16-\frac{25}{2} \frac{b \delta_{e}^{2}}{T^{2}}\right),
\end{aligned}
$$

so that the ellipticity is again the driving force for the nematic transition. However, in this case $\lambda_{\phi}^{3 p_{-}}$(which always becomes negative first) changes sign only below a temperature $T^{*}$ scaling as $T^{*} \sim 0.19 \delta_{e}$ [37]. At the same time the good orbital nesting pushes the magnetic transition to higher temperatures, since $\Pi_{X / Y}^{y z / x z} \sim 3 \Pi_{e h} / 8$.

To make a quantitative comparison between the two threepocket models, we show in Figs. 3(a) and 3(b) the magnetic susceptibility $\chi_{X / Y}^{y z / x z}(q=0)$ and the nematic eigenvalue $\lambda_{\phi}$ using the same set of band parameters, as appropriate, e.g., for 122 compounds [37]. As one can see, by accounting uniquely for the different orbital nesting the Néel temperature of the $3 p_{+}$model, $T_{\text {Néel }}^{3 p_{+}}$, is suppressed by about $80 \%$ with respect to the $3 p_{-}$case. Taking into account also that the experimental density of states in FeSe is smaller than in 122 compounds [18], $T_{\text {Néel }}^{3 p_{+}}$is expected to be further suppressed [37]. Finally from Fig. 3(b), one observes that while $\lambda_{\phi}^{3 p_{+}}$is always negative, $\lambda_{\phi}^{3 p_{-}}$changes sign slightly above the $T_{\text {Néel }}^{3 p_{-}}$, and then rapidly increases in absolute value. These considerations provide a possible explanation of the observed proximity between the nematic and magnetic transition in 122 systems [41].

The above results offer also a possible explanation for the suppression of nematicity in FeSe under internal and external pressure. Indeed, it has been reported that sulfur isoelectronic substitution [24,42] brings back the inner hole pocket above the Fermi level. This finding is also supported by $a b$ initio calculations, which usually miss the experimental position of the Fermi level but report in general an increase of the hole-pockets size with pressure [21,43]. The emergence of the inner hole pocket changes the FS topology of FeSe toward the more symmetric four-pocket model, which has been shown before to be detrimental for nematicity, leading to the largest positive value of the $\lambda_{\phi}^{0}$ eigenvalue. On the other hand, the same mechanism could also enhance magnetism, as observed. How these two effects interplay with the concomitant increase of the superconductivity remains an open question for future studies.

In conclusion, we derived the effective model for the spin fluctuations starting from a multiorbital low-energy fourpocket fermionic model. We showed that orbital degrees of freedom renormalize the effective interactions between spin modes, with observable consequences on the magnetic and nematic instabilities. We considered explicitly a prototype three-pocket model, as appropriate for FeSe and 122 compounds, where the only difference between the two cases is the orbital content of the relevant $h$ pocket at $\Gamma$. In FeSe the orbital mismatch between the outer $\mathrm{h}$ pocket and the electron ones boosts nematicity and is detrimental for magnetism. In 122 compounds the good h-e orbital nesting favors magnetism and makes nematicity possible only at temperatures close to the magnetic transition. Our results offer a unified scenario to understand how orbital nesting can differentiate topologically equivalent band structures. Further confirmations of this mechanism can provide a useful tool to ultimately reach the external control on nematic order in iron-based systems.

L.F. and B.V. acknowledge Jörg Fink for useful discussions. B.V. acknowledges Roser Valenti for discussions and for sharing her $\mathrm{ab}$ initio calculations of FeSe with pressure. L.B. acknowledges financial support by Italian MIUR under project PRIN-RIDEIRON-2012X3YFZ2 and by MAECI under the Italian-India collaborative project SUPERTOP-PGR04879. B.V. acknowledges funding from Spanish MINECO (Spain) via Grant No. FIS2014-53219-P and Fundación Ramón Areces.
[1] Y. Gallais and I. Paul, C. R. Phys. 17, 113 (2016).

[2] R. M. Fernandes, A. V. Chubukov, J. Knolle, I. Eremin, and J. Schmalian, Phys. Rev. B 85, 024534 (2012).

[3] R. M. Fernandes, A. V. Chubukov, and J. Schmalian, Nat. Phys. 10, 97 (2014).
[4] A. E. Böhmer, F. Hardy, F. Eilers, D. Ernst, P. Adelmann, P. Schweiss, T. Wolf, and C. Meingast, Phys. Rev. B 87, 180505 (2013).

[5] S. Baek, D. Efremov, J. M. Ok, J. S. Kim, J. van den Brink, and B. Büchner, Nat. Mater. 14, 210 (2014). 
[6] Y. Su, H. Liao, and T. Li, J. Phys.: Condens. Matter 27, 105702 (2015).

[7] S. Mukherjee, A. Kreisel, P. J. Hirschfeld, and B. M. Andersen, Phys. Rev. Lett. 115, 026402 (2015).

[8] K. Jiang, J. Hu, H. Ding, and Z. Wang, Phys. Rev. B 93, 115138 (2016).

[9] L. Fanfarillo, G. Giovannetti, M. Capone, and E. Bascones, Phys. Rev. B 95, 144511 (2017).

[10] R.-Q. Xing, L. Classen, M. Khodas, and A. V. Chubukov, Phys. Rev. B 95, 085108 (2017).

[11] Q. Wang, Y. Shen, B. Pan, Y. Hao, M. Ma, F. Zhou, P. Steffens, K. Schmalzl, T. R. Forrest, M. Abdel-Hafiez et al., Nat. Mater. 15, 159 (2016).

[12] M. C. Rahn, R. A. Ewings, S. J. Sedlmaier, S. J. Clarke, and A. T. Boothroyd, Phys. Rev. B 91, 180501 (2015).

[13] L. Fanfarillo, A. Cortijo, and B. Valenzuela, Phys. Rev. B 91, 214515 (2015).

[14] M. H. Christensen, J. Kang, B. M. Andersen, and R. M. Fernandes, Phys. Rev. B 93, 085136 (2016).

[15] R. Fernandes and A. Chubukov, Rep. Prog. Phys. 80, 014503 (2017).

[16] S. Onari, Y. Yamakawa, and H. Kontani, Phys. Rev. Lett. 116, 227001 (2016).

[17] J. K. Glasbrenner, I. I. Mazin, H. O. Jeschke, P. J. Hirschfeld, R. M. Fernandes, and R. Valenti, Nat. Phys. 11, 953 (2015).

[18] L. Fanfarillo, J. Mansart, P. Toulemonde, H. Cercellier, P. Le Fèvre, F. Bertran, B. Valenzuela, L. Benfatto, and V. Brouet, Phys. Rev. B 94, 155138 (2016).

[19] A. V. Chubukov, M. Khodas, and R. M. Fernandes, Phys. Rev. X 6, 041045 (2016).

[20] Y. Yamakawa, S. Onari, and H. Kontani, Phys. Rev. X 6, 021032 (2016).

[21] Y. Yamakawa and H. Kontani, Phys. Rev. B 96, 144509 (2017).

[22] J. P. Sun, K. Matsuura, G. Z. Ye, Y. Mizukami, M. Shimozawa, K. Matsubayashi, M. Yamashita, T. Watashige, S. Kasahara, Y. Matsuda et al., Nat. Commun. 7, 12146 (2016).

[23] K. Kothapalli, A. E. Böhmer, W. T. Jayasekara, B. G. Ueland, P. Das, A. Sapkota, V. Taufour, Y. Xiao, E. Alp, S. L. Budko et al., Nat. Commun. 7, 12728 (2016).
[24] A. I. Coldea and M. D. Watson, Ann. Rev. Cond. Matt. Phys. 9, 125 (2018).

[25] M. Khodas and A. Levchenko, Phys. Rev. B 91, 235119 (2015).

[26] M. H. Christensen, J. Kang, B. M. Andersen, I. Eremin, and R. M. Fernandes, Phys. Rev. B 92, 214509 (2015).

[27] L. Classen, R.-Q. Xing, M. Khodas, and A. V. Chubukov, Phys. Rev. Lett. 118, 037001 (2017).

[28] Y. Ran, F. Wang, H. Zhai, A. Vishwanath, and D.-H. Lee, Phys. Rev. B 79, 014505 (2009).

[29] K. Kuroki, H. Usui, S. Onari, R. Arita, and H. Aoki, Phys. Rev. B 79, 224511 (2009).

[30] S. Graser, T. Maier, P. Hirschfeld, and D. Scalapino, New J. Phys. 11, 025016 (2009).

[31] A. F. Kemper, M. M. Korshunov, T. P. Devereaux, J. N. Fry, H.-P. Cheng, and P. J. Hirschfeld, Phys. Rev. B 83, 184516 (2011).

[32] E. Bascones, B. Valenzuela, and M. J. Calderón, C. R. Phys. 17, 36 (2016).

[33] V. Cvetkovic and O. Vafek, Phys. Rev. B 88, 134510 (2013).

[34] J. Fink, A. Charnukha, E. D. L. Rienks, Z. H. Liu, S. Thirupathaiah, I. Avigo, F. Roth, H. S. Jeevan, P. Gegenwart, M. Roslova et al., Phys. Rev. B 92, 201106 (2015).

[35] J. Fink, E. D. L. Rienks, S. Thirupathaiah, J. Nayak, A. van Roekeghem, S. Biermann, T. Wolf, P. Adelmann, H. S. Jeevan, P. Gegenwart et al., Phys. Rev. B 95, 144513 (2017).

[36] I. Avigo, S. Thirupathaiah, E. D. L. Rienks, L. Rettig, A Charnukha, M. Ligges, R. Cortes, J. Nayak, H. S. Jeevan, T. Wolf et al., Phys. Status Solidi B 254, 1600382 (2017).

[37] See Supplemental Material at http://link.aps.org/supplemental/ 10.1103/PhysRevB.97.121109 for further details.

[38] H. Ding, P. Richard, K. Nakayama, T. Sugawara, T. Arakane, Y. Sekiba, A. Takayama, S. Souma, T. Sato, T. Takahashi et al., Europhys. Lett. 83, 47001 (2008).

[39] N.-L. Wang, H. Hosono, and P. Dai, Iron Based Superconductors (Pan Stanford Publishing, USA, 2013).

[40] R. M. Fernandes and J. Schmalian, Supercond. Sci. Technol. 25, 084005 (2012).

[41] I. Paul, Phys. Rev. B 90, 115102 (2014).

[42] P. Reiss, M. D. Watson, T. K. Kim, A. A. Haghighirad, D. N. Woodruff, M. Bruma, S. J. Clarke, and A. I. Coldea, Phys. Rev. B 96, 121103 (2017).

[43] R. Valenti (private communication). 